\title{
A parameter study of Pythia6 MPI model using LHC data
}

\author{
N. Firdous \\ GIFT University, Pakistan \\ E-mail: nameeqa@gift.edu.pk \\ M. Junaid \\ GIFT University, Pakistan

\section{G. Rudolph} \\ University of Innsbruck, Austria
}

\begin{abstract}
PYTHIA is a well established Monte Carlo event generator developed over the past several decades with the experimental discoveries. The core of PYTHIA model for soft hadronic interactions is based on a phenomenological adaptation, such as Multiple Parton Interaction (MPI) model, to describe the non perturbative pp processes. A parameter study of this MPI model using best fit to the data published by ATLAS Collaboration is presented. Altogether thirteen parameters have been investigated to find the most sensitive parameters to the selected data. This type of study helps in better understanding and selection of sensitive parameters needed to be considered in PYTHIA6 tunes.
\end{abstract}

38th International Conference on High Energy Physics

3-10 August 2016

Chicago, USA 


\section{Introduction}

Monte Carlo (MC) event generators such as PYTHIA, PHOJET and HERWIG etc., are used to make predictions for collider experiments. Different phenomenological models are implemented in these event generators with many free parameters which need to be tuned to improve the agreement with the data. In this paper PYTHIA6 [1] is considered and the optimization of its model parameters is presented. This work mainly focuses on study of tuned parameters related to Multiple Parton Interaction Model, published Minimum Bias data from ATLAS [3] at 0.9 and $7 \mathrm{TeV}$ is used. The method employed to tune the parameters is based on a linear and iterative approach [2] and allows the simultaneous variation of many parameters. Therefore, thirteen parameters of different models were considered for tuning. This workisthe continuation of [4].

In the following section the parameter study is described to see the impact of these parameters on the selected observables.

\section{Parameters Correlation}

At the end of $\chi^{2}$ minimization Minuit calculates the correlation between all the tuning parameters using the covariance matrix. Correlation coefficients are given in Table 1. The strongest correlation exists between $p_{t 0}(\operatorname{PARP}(82))$ and $\Lambda_{M E}(\mathrm{PARP}(1))$. The next strong correlation is observed between color reconnection parameters, PARP(78) and PARP(77). Remaining parameters are less correlated. This type of study helps to understand the behavior of the free parameters. It has been observed that any strong correlation between parameters sometimes causes an instability.

\section{Relative Accuracy}

Taking the correlation into account, Minuit software provides error estimates for each fitted parameter. The ratio of error divided by value (relative error) is an indication of the accuracy of the parameter determination.

Table 2 contains relative error of thirteen parameters. It is clear from the table that UE/MPI model parameters $p_{t 0}$ and energy evolution have small relative error which means they are well constrained/determined by the data used. The same is true for the matter overlap parameter. $\Lambda_{Q C D}$ parameters, PARP(72) and PARP(1), also have small relative error so treated as adjustable parameters. $\Lambda_{Q C D}$ parameter for ISR, PARP(61), has larger relative error as compared to other two $\Lambda_{Q C D}$ parameters. Color reconnection model parameters have large errors, but this is because of their strong positive correlation between them as listed in Table 1. Sensitivity study shows that these parameters are very sensitive to $p_{t}$ and $\left\langle p_{t}\right\rangle v s$. Nch distributions. We treated only PARP(78) as an adjustable parameter in final tunes.

Parameters related to the beam remnant have much higher error as compared to other parameters, which means that these parameters can not be well constrained by the selected data. So these parameters are kept constant at best fit values obtained from preliminary tunes. 


\begin{tabular}{crrrrrrr}
\hline PARP & 82 & 83 & 78 & 77 & 1 & 72 & 90 \\
\hline 82 & 1 & -0.589 & 0.087 & 0.294 & 0.949 & -0.030 & -0.274 \\
83 & & 1 & -0.010 & -0.141 & -0.446 & -0.418 & 0.021 \\
78 & & & 1 & 0.871 & 0.133 & -0.203 & -0.365 \\
77 & & & & 1 & 0.35 & -0.081 & -0.404 \\
1 & & & & & 1 & -0.167 & -0.420 \\
72 & & & & & & 1 & 0.156 \\
90 & & & & & & & 1 \\
\hline
\end{tabular}

Table 1: Correlation coefficients for seven MPI parameters as calculated by Minuit

\begin{tabular}{lcc}
\hline Parameter & Type & Relative error\% \\
\hline PARP(82) & UE & 0.41 \\
PARP(90) & UE & 0.25 \\
PARP(83) & UE & 0.33 \\
PARP(72) & IFSR & 1.1 \\
PARP(71) & IFSR & 13.0 \\
PARP(1) & ME & 1.5 \\
PARP(61) & ISR & 2.6 \\
PARP(67) & ISR & 15.0 \\
PARP(91) & BR & 2.1 \\
PARP(79) & BR & 6.3 \\
PARP(80) & BR & 9.7 \\
PARP(78) & CR & 2.7 \\
PARP(77) & CR & 2.5 \\
\hline
\end{tabular}

Table 2: Relative error

\section{Stability of Parameters w.r.t Distributions}

It is important to study the stability of the minimum found with respect to the distributions of selected data sets from ATLAS, CDFII and CMS. Altogether eight parameters are fitted using different combinations of the observables at three different energies: $0.9 \mathrm{TeV}, 1.9 \mathrm{TeV}$ and $7 \mathrm{TeV}$. While devising the combinations of observables we ensure that parameter sensitivity is not compromised. Figure 1 shows the fitted parameter values with respect to the $\chi^{2}$ values obtained from different observable combinations. It can be seen that PARP(90) and PARP $(83)$ are most stable parameters, whereas PARP(61) seems less stable as its values show more spread w.r.t different $\chi^{2}$ values.

\section{Conclusion}

Altogether thirteen parameters of selected physics models offered by PYTHIA6 are investigated using best fit to ATLAS MB data at two energies. This study improves the understanding of MPI model parameters and their behaviour at different energies, relative acuracy. Important correlations existed between selected parameters are also discussed. 

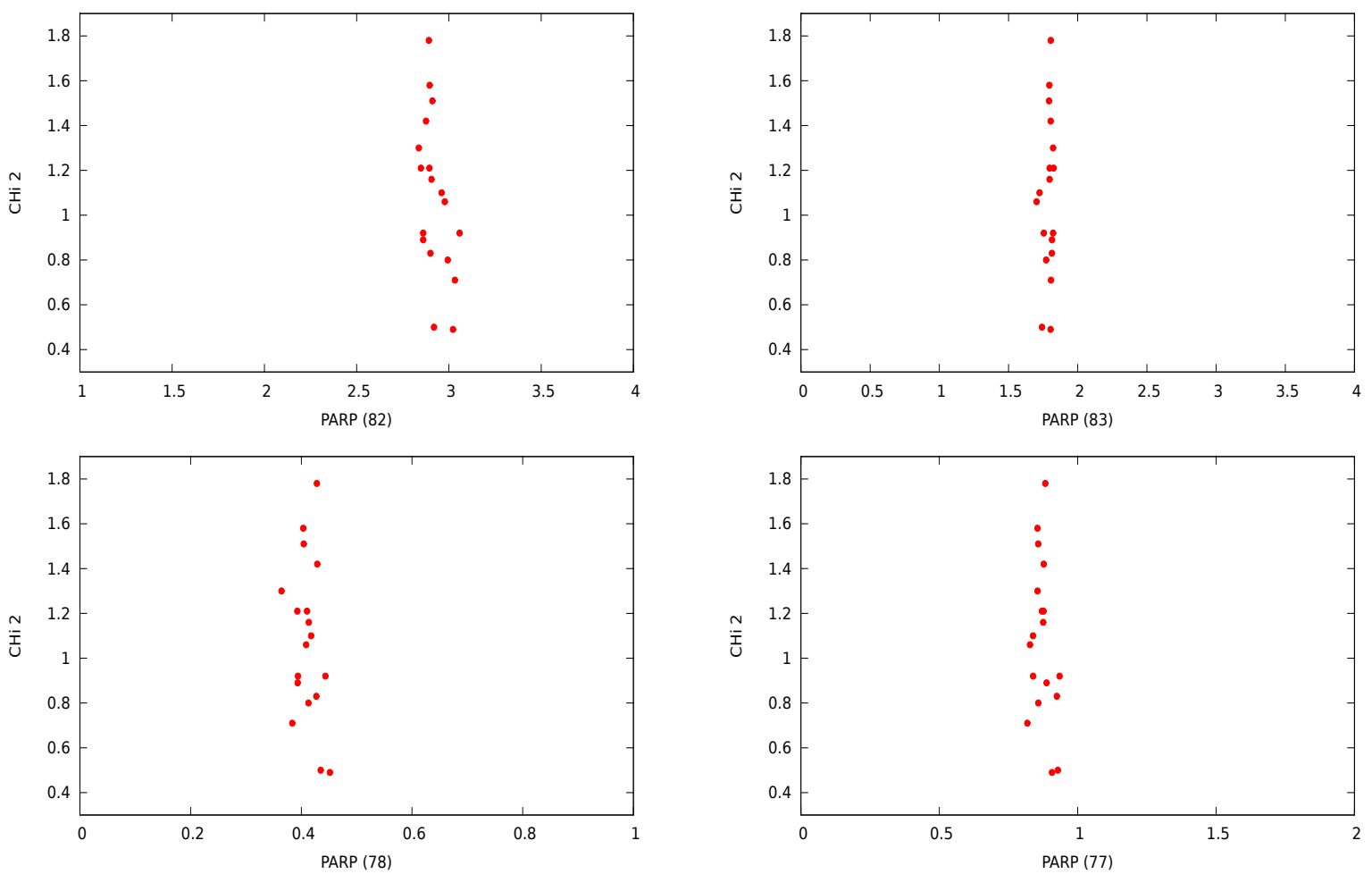

Figure 1: Stability plots of eight parameters w.r.t $\chi^{2} / N_{d f}$ obtained from different distribution combinations

\section{References}

[1] Torbjörn Sjöstrand, Stephen Mrenna, and Peter Z. Skands. PYTHIA 6.4 Physics and Manual.JHEP, 05:026, 2006.

[2] R. Barate et al. Studiesof quantum chromodynamics with the ALEPH detector. Phys.Rept., 294:1-165, 1998.

[3] G. Aad et al. Charged-particle multiplicities in pp interactions measured with the ATLAS detector at the LHC.New J.Phys., 13:053033, 2011.

[4] Nameeqa Firdous. Gerald Rudolph. Investigation of phenomenological models implemented in Pythia6 .EJP Web of Conferences, 60, 20009, 2013. 